\title{
APRESENTAÇÃO PRIMEIRA EDIÇÃO ESPECIAL: EDUCAÇÃO E DEMOCRACIA EM TEMPOS DE PANDEMIA
}

\author{
Márcia Denise Pletsch ${ }^{\mathrm{i}}$ \\ Izadora Martins da Silva deSouzaii \\ Saionara Corina Pussenti Coelho Moreira ${ }^{\text {iii }}$ \\ Alexandre Rodrigues Assis ${ }^{\text {iv }}$
}

"Um dia de cada vez..."

A década de 2020 começou de maneira i-nes-pe-ra-da! Com a descoberta do novo coronavírus muitas coisas mudaram e se transformaram. De acordo com os informativos, a doença se originou na cidade chinesa de Wuhan no ano de $2019^{\mathrm{v}}$. O vírus se alastrou pelo mundo ocasionando mortes e consequências desconhecidas à saúde da população. O reconhecimento da complexidade levou a Organização Mundial da Saúde (OMS) a declarar em 11 de março de 2020 o estado de pandemia ${ }^{\text {vi }}$. Até o presente momento ainda aprendemos a lidar com a doença e com suas múltiplas consequências econômicas, políticas, sociais e educacionais.

Passado-se meses das primeiras medidas de contenção da pandemia da covid-19 no Brasil, contemplamos um cenário de incertezas diante do "novo normal". Caracterizado como conceito de mudança para o bem comum, o "novo" e o "normal" se tornam relativos, já que evidenciam um padrão inexistente em uma sociedade de múltiplos contextos. Certamente a pandemia aligeirou a excessiva dependência no uso dos artefatos digitais, configurando o "superhumano" no seu espaço home office, como também o isolamento social prosperou a indústria de laboratórios virtuais. O desafio de compreender o "novo normal” indica uma percepção além da crise de saúde pública, mas precisamos olhar a rede inteira do contexto. A pandemia exacerba desigualdades, sejam elas sociais ou educacioanis. 
Março de 2020 foi marcado pela hashtag \#movimentoficaemcasa que ampliou-se diante do cenário alarmante com centros hospitalares que não comportavam a demanda, o déficit na saúde ficou mais explícito, o que implicou em novas sanções. Diante dos riscos produzidos no contexto econômico-social, ocorreram discussões de como dar assistência aos trabalhadores informais, desempregados e autônomos no contexto da pandemia. O Senado aprovou o projeto de lei do auxílio emergencial no valor de $\mathrm{R} \$ 600,00$. Este foi noticiado por ocasionar aglomerações de pessoas na tentativa de recebimento do auxílio.

Além das iniciativas para desenfrear a letalidade do vírus e a crise social, outro enfrentamento foi em relação à fake news. Muitas notícias compartilhadas de fontes inseguras, gerando descuidado a respeito da saúde pública foram/são distribuídas pela internet. A veiculação de notícias falsas não é um fenômeno restrito ao período da pandemia, mas ocorreu a intensificação na produção de conteúdo sensacionalista. Sobre isso, a Fundação Oswaldo Cruz (FIOCRUZ) conduziu um estudo que apontou as principais plataformas digitais usadas para o compartilhamento de informações adulteradas. Com o apoio de um aplicativo chamado "Eu fiscalizo" foi realizado um levantamento por amostragem que identificou o Instagram, Facebook e WhatsApp como as mais utilizadas para compartilhamento de fake news em relação à covid-19 $9^{\text {vii }}$.

O novo coronavírus nos antecipou algumas afirmativas. Vivendo entre a vida e a morte e divergências de mundo, a pandemia tem diferentes faces: o negacionismo da ciência que exacerba o quantitativo de vítimas; a corrupção que reduz as chances de manutenção da vida; as desconsiderações de medidas e recomendações da ciência demonstram irresponsabilidade.

A oposição frente ao inimigo invisível ocasionou a suspensão das aulas nas instituições de ensino, em diferentes níveis. Ocorreu a necessidade de alternativas educacionais, como proposta o uso de tecnologias digitais para possibilitar a interação entre instituições e estudantes. A implementação de propostas pedagógicas por infraestruturas de conexões e dependência da internet começaram por diferentes nomenclaturas e orientações a respeito do ensino não-presencial. O poder público assentiu ações autônomas de cada estado/município e documentos distintos de acordo com suas secretarias de educação. Porém é importante investigar os discursos e os recursos de midiatização que foram disponibilizados.

A proposta de continuidade das atividades pedagógicas ocasionou diferentes opiniões entre especialistas e fortes pressões de interesses privado. Em meio às considerações de encerramento do ano letivo na educação e o receio do abandono escolar, a pandemia evidencia o tamanho do abismo social na sociedade da informação e do conhecimento. Entre o uso de apostilas e interatividade em diferentes plataformas pagas/gratuitas, o ensino remoto ganhou 
repercussão e revelou disparidades quanto ao acesso à tecnologia digital em diferentes âmbitos. As redes de comunicação fomentam argumentos de uma sociedade aparelhada por artefatos digitais, firmando as gerações recentes como detentoras desses apetrechos, o que se considera nativismo digital. Entretanto, o aumento do uso da tecnologia digital nesse período retrata desafios em relação à acessibilidade, usabilidade e igualdade de direitos. Muitos estudantes não participam efetivamente das propostas pedagógicas de atividades remotas, pois há discrepância quanto ao acesso à internet.

Destacamos o relatório Inclusion and education: All means all, idealizado no ano de 2020 pela Organização das Nações Unidas para a Educação, a Ciência e a Cultura (UNESCO). Ele apresenta informações sobre a inclusão e o contraste vivido nos cenários educacionais pelo mundo. O documento revelou que $40 \%$ de mais de 200 países pesquisados não têm condições de oferecer o ensino remoto no período da pandemia, entre eles o Brasil se destaca pelas desigualdades educacionais.

Nesse contexto, o Conselho Nacional de Educação (CNE) no dia 06 de outubro de 2020 aprovou uma série de resoluções e normas orientativas para escolas públicas e privadas do país. Entre essas recomendações o texto propõe e permite que o ensino remoto continue até 31 de dezembro de 2021. As instituições de ensino superior pública e privada também receberam orientações para substituir as aulas presenciais para as aulas on-line até o final de 2021. O CNE é responsável por estabelecer normas educacionais às instituições de ensino, conforme a Lei $\mathrm{n}^{\circ}$ 14.040/2020. Ele é um dos órgãos mais importante dentro do sistema educacional brasileiro, é formado por 12 conselheiros indicados pelo presidente da República e é responsável por diagnosticar problemas e assessorar no Plano Nacional de Educação e especialmente dar suporte a Ministério da Educação (MEC), o conselho emite ainda pareceres e resoluções que devem ser seguidos pela União, estados e municípios após a homologação do MEC.

Compreendendo a educação como um direito humano, observa-se que a década de 2020 reacende urgências de debates quanto ao direito à educação e a respeito das tecnologias educacionais. Falar do campo da educação requer um olhar alongado, já que nada nesse espaço pode ser considerado intuitivo. Nesse sentido, o acesso e a participação de todos expõem diálogos de conflitos, desigualdades e forças de interesse.

Para refletir sobre algumas dessas questões a presente edição apresenta um conjunto de artigos de autores de diferentes regiões do Brasil e de Portugal, os quais analisam possibilidades e apresentam dados sobre o momento vivido da pandemia da covid-19, perpassando por diferentes modalidades e níveis de ensino.

Os artigos e relatos de experiência, assim como uma resenha que compõem esta 
Edicação Especial reafirmam a pertinência do estudo da temática. A Edição Especial I: Educação e Democracia em Tempos de Pandemia, que integra o volume 6 de 2020, é organizado pela Professora Doutora Márcia Denise Pletsch que é coordenadora geral do Observatório de Educação Especial e Inclusão Educacional (ObEE) da Universidade Federal Rural do Rio de Janeiro (UFRRJ), pela Pedagoga e Mestre Izadora Martins da Silva de Souza integrante do ObEE/UFRRJ e do Grupo de Pesquisa Discursos da Educação e Tecnologia (DEdTec) da PUC-Rio, pela Professora Mestre Saionara Corina Pussenti Coelho Moreira da Rede Municipal de Educação de Nova Iguaçu e integrante do ObEE/UFRRJ e pelo Professor Doutor Alexandre Rodrigues Assis da Rede Estadual de Educação do Rio de Janeiro e integrante do Grupo de Estudos e Pesquisas das Tecnologias da Informação e Comunicação (TIC) em Educação Matemática (Gepeticem/UFRRJ) e pela Professora Mestre Saionara Corina Pussenti Coelho Moreira da Rede Municipal de Educação de Nova Iguaçu e integrante do ObEE/UFRRJ.

O primeiro artigo desta edição "Ensino remoto e as contradições no trabalho docente" faz alusão às práticas de cinco professores do ensino privado e público, que através de entrevistas semiestruturadas elucidaram como foram orientados a colocar em prática as atividades de ensino remoto durante a pandemia, as entrevistas foram aplicadas no modo online. O destaque para o uso da Teoria da Atividade (LEONTIEV, 2004) dando ênfase ao trabalho coletivo e uso de instrumentos e a linguagem.

Carolina Alves de Oliveira e Léa Tiriba apresentam a valorização do cuidar das questões de gênero, destacando mulheres de classes populares no trabalho intitulado "Pandemia, crise de cuidados e educação ambiental: o que aprender com quem cuida da vida?".

$\mathrm{O}$ artigo intitulado "Impactos do covid-19: as visões e experiências de crianças e jovens portugueses em situação de acolhimento residencial" apresenta uma realidade de crianças e jovens vivendo em uma instituição de acolhimento residencial em Portugal e ressalta que a pandemia apresentou a importância da preparação dessa instituição para garantir que essas pessoas tenham acesso garantido aos momentos de aprendizagem através das atividades online. Participaram da pesquisa 9 jovens de 11 a 21 anos, e além da escola eles frequentavam antes da pandemia cursos profissionais (cabeleireiro, restauração e cozinha).

O texto de Cordeiro e Costa intitulado "Educação na pandemia do novo coronavírus: mídias e desigualdade" aponta a falta estrutural e a desigualdade no país em relação ao acesso às mídias. Em destaque para a questão do uso de tecnologias da comunicação nos domicílios brasileiros, e a acentuada falta de acessibilidade no uso das TICS e acesso ao ensino remoto.

$\mathrm{O}$ presente artigo "Novos espaços de afeto e cuidado em tempos de isolamento 
social" narra a trajetória de um projeto presencial aplicado aos alunos de uma escola pública, que com o advento da pandemia precisou ser estruturado para uma nova abordagem on-line, com a aprovação dos alunos em 95\%. E, dessa forma, o projeto deu continuidade às práticas educativas oriundas do projeto "cuide-se".

No artigo de Marcio Caetano, Paulo Melgaço Silva Junior e Tarciso Manfrenatti de Souza Teixeira, intitulado "Educação em tempos de pandemia: reflexões sobre políticas de educação na cidade do Rio de Janeiro", são apresentadas percepções docentes em relaçãoàs propostas da Secretaria Municipal e as realidades desiguais ao acesso de recursos digitais por diversos estudantes.

O artigo "A exclusão e seus desdobramentos oriundos de uma pandemia: reflexões para a construção de uma escola inclusiva" relata a exclusão antes da pandemia e a mudança que poderá acontecer pós-pandemia em relação aos alunos com deficiência e os avanços que todo esse movimento atual irá provocar para esse público. O artigo de autoria de Vivian Martins, Bárbara Rodrigues de Castro e Michelle Viana Trancoso, "Criações e percepções docentes no ensino remoto durante a pandemia de covid-19: uma pesquisa com os cotidianos", mostra a narrativa e escrita docente, analisando uma determinada rede social.

O texto "Processos formativos e extensão universitária: as ações da quarentena em foco", de autoria de Mônica Maria Farid Rahme, Ana Maria Fernandes Viana e Deborah Stephanie de Oliveira Henrique, analisa o trabalho de extensão, possibilitando reflexões da parceria entre universidade e a comunidade, explorando espaços de trabalho educativo em comprometimento com a democracia e com a produção de saberes.

A autora Tamara França de Almeida Magalhães em seu artigo "Escolarização do estudante com deficiência em tempos de pandemia da covid-19: tecendo algumas possibilidades" relata o processo de escolarização dos alunos com deficiência durante o período de isolamento social e todas as suas implicações, destaca pontos importantes como desigualdades sociais e a necessidade de políticas públicas na área, e que contemplem a pessoa com deficiência.

Aline de Carvalho Moura e Andreia Gomes da Cruz no seu artigo "Ensino superior e produtividade acadêmica em tempos de pandemia" traz uma pesquisa voltada para o levantamento bibliográfico e análise documental que relatam as ações do docente no ensino superior no cenário da covid-19, a questão da produtividade é enfatizada no decorrer da escrita.

“Onde estão os bebês nas políticas? governar a infância para governar a vida" apresenta questionamentos que instigam o leitor a caminhar sobre o texto, para entender o lugar que esse público deve estar e ser inserido. As políticas públicas devem ser voltadas para a criação 
de creches e espaços que acolham os bebês e com isso orientações que permeiam a vida do bebê como parte integrante de uma sociedade e ganhe mais visibilidade em seus direitos e a defesa de ser e estar no mundo.

No artigo seguinte "A pandemia do coronavírus e os efeitos na educação: reflexões em curso", Roberta de Mendonça Porto e Jéssica Coelho de Lima Pereira analisam a partir de Michel Foucault os efeitos da pandemia da covid-19 na educação e experiências vividas durante o contexto de isolamento.

Em "A arte de reinventar a educação e o papel da cibercultura em tempos de distanciamento social" Leila Santos de Santana, Lucia Helena de Andrade Santos, Luciana Velloso da Silva Seixas e Tamires Elaine Barbosa Reis desenvolvem um estudo sobre as situações educativas ocasionadas pela pandemia, identificando dispositivos na lei e o uso das tecnologias digitais na continuidade das atividades escolares no presente contexto.

O Relato de experiência "Educação de pessoas surdas em tempos de pandemia: linguagem e relações de poder" das autoras Jéssika Figueredo Alves e Jacqueline de Souza Gomes permite vislumbrar os percalços construídos durante a pandemia na educação dos surdos de uma escola pública e as ações dos professores e intérpretes para que os alunos continuassem suas atividades remotas durante a pandemia.

Fabiana Pessanha e Nayara Alves Macedo discutem a "Educação da pequena infância: (re)pensando limites e possibilidades diante de algumas inflexões impostas pela pandemia da covid-19". Contribuindo para um exercício reflexivo a respeito dos Estudos da Infância, as autoras dialogam com seus estudos e experiências, provocando reflexões acerca do fechamento das instituições escolares.

Em seguida o trabalho "Nova gestão pública e redes de governança como sentido na gestão da educação tocantinense nas circunstâncias da pandemia", de Leonardo Victor dos Santos, Rosilene Lagares, Meire Lúcia Andrade da Silva, apresenta o Sistema de Ensino do Tocantins quanto às orientações para a Rede Pública no contexto da pandemia, observando disputas entre empresariado e o campo da educação.

Luis Henrique Monteiro de Castro e Rosemary dos Santos apresentam no artigo intitulado "Cambiências formativas em tempo de novas educações: o que aprendemos ensinamos com a pandemia" a investigação e o mapeamento de práticas de ensino desenvolvidas em um ambiente virtual de aprendizagem (AVA) e na multiplataforma de mensagens Whatsapp.

$\mathrm{O}$ artigo "A educação de jovens e adultos em tempos de pandemia: contradições e racionalidades em evidência", de autoria Dayana de Oliveira Arruda, Antônio Carlos do 
Nascimento Osório e Sara Santana Armoa da Silva, problematiza discursos e acontecimentos impostos pela pandemia de covid-19 na modalidade de Educação de Jovens e Adultos (EJA).

A resenha "Potencialidades das humanidades para a construção de uma sociedade democrática" de Adilson Cristiano Habowsk apresenta a obra Sem fins lucrativos: por que a democracia precisa das humanidades. O livro é da filósofa norte-americana Martha Craven Nussbaum que expõe a crise global da educação humanista e o seu dano para o futuro da democracia.

Por fim, entendemos que o desenvolvimento dos artigos se entrelaçam nas suas diversificadas abordagens sobre o tema, que perpassam a sala de aula do ensino básico, instituições de ensino superior, casas de acolhimento, e também o modo de vida de vários atores que compõem a educação antes, durante e pós-pandemia. Assim, esperamos que este Edição Esecial composto por várias pesquisas, com dados científicos coletados por autores comprometidos com uma sociedade plural, possam despertar nos leitores um olhar atento e curioso. Para as reflexões apresentadas, desejamos que se tornem referências para outros estudos científicos.

Boa leitura!

\section{Referência}

UNESCO. 2020. Global Education Monitoring Report 2020: Inclusion and education: All means all. Paris, UNESCO. Disponível em: https://en.unesco.org/gemreport/taxonomy/term/237. Acesso em: 19 out. 2020.

\footnotetext{
${ }^{i}$ Doutora em Educação, com pós-doutorado na Goethe Universität (Frankfurt/Alemanha) e na Universidade do Estado de Santa Catarina (Udesc). Mestre em Educação e Especialista em Altas Habilidades pela Universidade do Estado do Rio de Janeiro (UERJ). Graduada em Educação Especial, com Habilitação em Deficiência Intelectual, pela Universidade Federal de Santa Maria (UFSM). Professora Associada do Instituto Multidisciplinar, do Programa de Pós-Graduação em Educação, Contextos Contemporâneos e Demandas Populares (PPGEduc/UFRRJ) e do Programa de Pós-Graduação em Humanidades Digitais da Universidade Federal Rural do Rio de Janeiro (PPGIHD/UFRRJ). É coordenadora do Núcleo de Inclusão e Acessibilidade (NAI) da UFRRJ e do Observatório de Educação Especial e Inclusão Educacional (ObEE/UFRRJ), que integra o Centro de Inovação Tecnológica e Educação Inclusiva (CITEI) da UFRRJ - Campus de Nova Iguaçu. Riode janeiro, Brasil. E-mail: marciadenisepletsch@gmail.com ORCID: https://orcid.org/0000-0001-5906-0487

ii Doutoranda e mestre em Educação pelo Programa de Pós-Graduação em Educação, Contextos Contemporâneos e Demandas Populares da Universidade Federal Rural do Rio de Janeiro (PPGEduc/UFRRJ). Bolsista CAPES. Especialista em Tecnologias e Educação a Distância pela Faculdade São Luiz. Graduada em Pedagogia pela UFRRJ. Integrante do Grupo de Pesquisa Observatório de Educação Especial e Inclusão Educacional (ObEE/UFRRJ). Participa do Grupo de Pesquisa Discursos da Educação e Tecnologia (DEdTec), ligado ao Departamento de Educação da Pontifícia Universidade Católica do Rio de Janeiro (PUC-Rio). É editora executiva na Revista Interinstitucional Artes de Educar - RIAE. Desenvolve pesquisas em Educação Inclusiva; Tecnologia; Desenho Universal para Aprendizagem. Rio de Janeiro, Brasil. E-mail: izadorasouza15@gmail.com ORCID: https://orcid.org/0000-0003-2760-4659.

iii Mestre em Educação pelo Programa de Pós-Graduação em Educação, Contextos Contemporâneos e Demandas
} 
Populares da Universidade Federal Rural do Rio de Janeiro (PPGEduc/UFRRJ). Especialista em Libras pela Faculdade São Luiz, Graduada em Pedagogia pela UFRRJ. Pesquisadora FAPERJ de Treinamento e Capacitação Técnica (TCT)-nível Mestre, e integrante do grupo de pesquisa Observatório de Educação Especial e Inclusão Educacional (ObEE/UFRRJ). Professora da Rede Municipal de Nova Iguaçu. Desenvolve pesquisas em Educação Inclusiva; Inclusão no Ensino Superior; Intersetorialidade, Saúde e Educação na pesquisa da Síndrome congênita do Vírus Zika (SCVZ). Rio de Janeiro, Brasil. E-mail: saionara.pussente@gmail.com ORCID: https://orcid.org/0000-0002-3618-2155.

iv Doutor em Educação pelo Programa de Pós-Graduação em Educação, Contextos Contemporâneos e Demandas Populares da UFRRJ (PPGEduc/UFRRJ) e Mestre pelo mesmo programa. Possui graduação em Matemática pela Universidade do Estado do Rio de Janeiro. Professor da Educação Básica - SEEDUC/RJ e Ensino Superior UNESA. Tem experiência na área de Formação de Professores dos Anos Iniciais do Ensino Fundamental. Curso de Especialização para Professores de Matemática pelo Projeto Fundão ( UFRJ) e Especialização em Novas Tecnologias no Ensino da Matemática ( LANTE - UFF). Desenvolve pesquisas na área de Educação Matemática, Tecnologia Digital Aplicada à Educação e Acessibilidade Digital. Interesse em temas como: semiótica, cognição corporificada e design de materiais pedagógicos. Integrante do GEPETICEM . Membro da equipe editorial do "Boletim de Educação Especial e Inclusão Escolar", da "Revista Ensaios e Pesquisas em Educação" e do GEPEM. Integrante do GT 6 da SBEM - Educação Matemática: Tecnologias Digitais e Educação a Distância. Rio de Janeiro, Brasil. E-mail: profalexandreassis@hotmail.com ORCID: https://orcid.org/0000-0002-3658-2829

v Informações acessadas na página da Fundação Oswaldo Cruz (FIOCRUZ). Disponível em: https://portal.fiocruz.br/. Acesso em: 10 out. 2020.

vi Disponível em https://www.unasus.gov.br/noticia/organizacao-mundial-de-saude-declara-pandemia-decoronavirus. Acesso em: 10 out. 2020.

vii A pesquisa faz parte do projeto de pós-doutoramento de Claudia Galhardi, supervisionada por Cecília Minayo e apoiado pela Fundação de Amparo à Pesquisa do Estado do Rio de Janeiro (Faperj). O aplicativo "Eu Fiscalizo" notifica aos usuários conteúdos impróprios nas plataformas digitais. Maiores informações em: http://informe.ensp.fiocruz.br/noticias/48662. 